\title{
Chapter
}

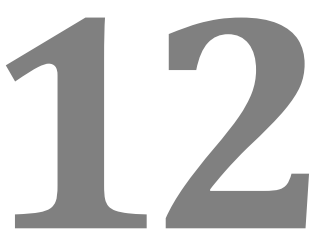

\section{A GENERAL OVERVIEW OF THE NANO-SIZED CARRIERS FOR CANCER TREATMENT}

Jeyshka M. Reyes-González ${ }^{1,2}$, Frances M. Pietri-Vázquez ${ }^{3}$, and Pablo E. Vivas-Mejía ${ }^{1,2 *}$

${ }^{1}$ Department of Biochemistry, University of Puerto Rico, Medical Sciences Campus, San Juan, Puerto Rico 00935, USA

${ }^{2}$ Comprehensive Cancer Center, University of Puerto Rico, Medical Sciences Campus, San Juan, Puerto Rico 00935, USA

${ }^{3}$ Medical School, University of Puerto Rico, Medical Sciences Campus, San Juan, Puerto Rico 00935, USA 


\section{Contents}

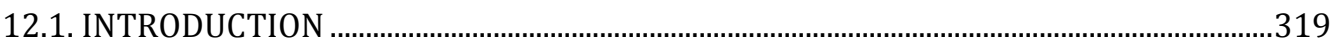

12.2. NANOTECHNOLOGY AND ITS DIVERSE APPLICATIONS ..................................................319

12.3. NANOPARTICLES AND CANCER MEDICINE

12.3.1. Common nanoparticles with potential use for cancer treatment and

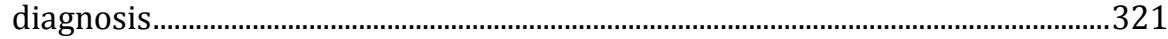

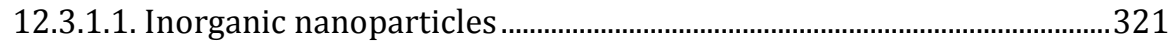

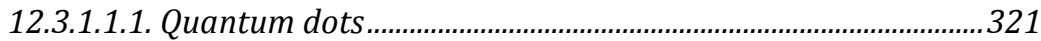

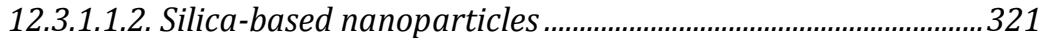

12.3.1.1.3. Metal-based nanoparticles............................................................322

12.3.1.1.4. Magnetic nanoparticles.............................................................322

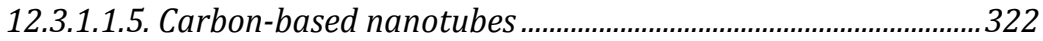

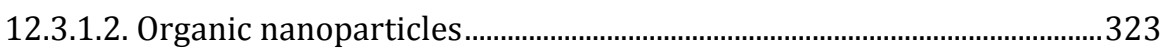

12.3.1.2.1. Polymer-based nanoparticles .................................................323

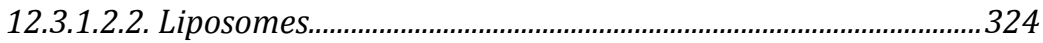

12.3.2. Nanoliposomes as delivery carriers of therapeutic and imaging agents

12.3.3. Nanoliposomes as delivery systems of siRNA and miRNAs for cancer

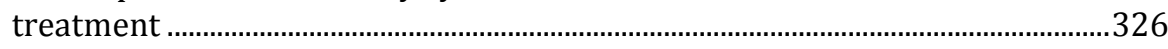

12.3.4. Other nanoparticles as delivery systems for therapeutic and imaging agents in cancer.

12.4. NANOPARTICLE FORMULATIONS FOR THE TREATMENT OF OTHER HUMAN CONDITIONS

12.5. FUTURE DIRECTIONS AND CHALLENGES .....................................................................

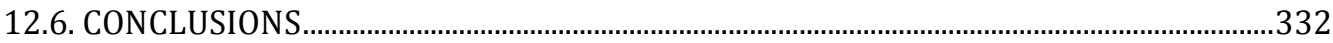

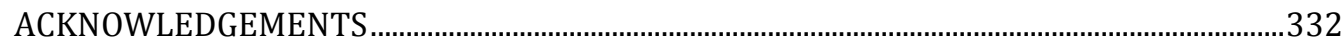

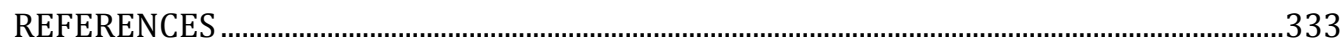




\subsection{INTRODUCTION}

Nanotechnology consists in the manipulation, creation, and use of substances in the nanometer scale for the fabrication of nanomaterials and nanodevices [1]. Nanotechnology has impacted diverse fields of research, including environmental science, cosmetics, the food industry, and medicine [2-5]. The major application of nanotechnology to medicine involves the creation of nanoparticles as delivery carriers for chemotherapeutic agents to prolong drug action and decrease side effects [1,2]. Although several nanomaterials have been proposed as drug carriers, nanoliposome-encapsulated drugs are the most used clinically [6]. In fact, several classic chemotherapeutic agents, including doxorubicin, daunorubicin, cytarabine, and vincristine, are now clinically used as nanoliposomal formulations [7-11]. The use of nanoliposomes for the delivery of emerging cancer therapies such as small interference RNA (siRNA) and microRNA (miRNA)-based molecules is also an exciting research field [6,12-14]. In this chapter, we will discuss the status of the nanoparticles currently used for cancer treatment and diagnosis. We place special emphasis on the use of nanoliposomes as drug carriers for novel cancer therapies. Finally, we discuss the major challenges of using nanoparticles as drug delivery systems for effective cancer treatment.

\subsection{NANOTECHNOLOGY AND ITS DIVERSE APPLICATIONS}

Nanotechnology is a novel field of research that develops materials and devices in a scale of 1-100 $\mathrm{nm}$. Having access to the nanoscale and the ability to develop nanomaterials has advanced various areas of research, including cosmetics, the food industry, environment-related sciences, and medicine.

In the food industry, nanotechnology has led to several advances in areas such as food packaging and food conservation. One example is the incorporation of silver nanoparticles (AgNPs) into packaging materials such as plastics, taking advantage of the antibacterial properties of silver [15]. AgNPs have also been integrated into a hydroxypropyl methylcellulose (HPMC) matrix, yielding increased tensile strength and barrier properties, and subsequently resulting in prolonged product quality and stability [16]. Nanotechnology has also been applied to assure greater stability, durability, bioavailability, and controlled release of bioactive food ingredients [17]. For example, zein - a storage protein found mainly in maize - is used to entrap, transport, and protect omega-3 polyunsaturated fatty acids such as docosahexaenoic acid (DHA), which has protective cardiovascular properties [17]. Zein has also been used to formulate 
curcumin-containing nanoparticles for the potential treatment of several illnesses, including cancer and Alzheimer's [18].

In the cosmetic industry, nanotechnology has been applied to many modern cosmetic products, such as sunscreen. For example, a study showed that using the clay montmorillonite (MMT) as a carrier of titanium-dioxide $\left(\mathrm{TiO}_{2}\right)$ - the major component of sunscreen products - yields a nanocomposite that enhances UV protection, and concomitantly increases the photostability and efficacy of sunscreen [19]. Also, nano-delivery systems that allow effective transport of compounds into the epidermis and deeper skin layers while keeping the biological properties of skin (such as moisture and squamous cell integrity) is an important area of research, especially for the ongoing development of topical creams and treatments. A recent study compared the use of three different nanocarriers - liposomes, solid lipid nanoparticles (SLNs), and nanoemulsions (NEs) - for the transport of retinoids (vitamin A derivatives used to treat skin conditions such as acne) [20]. The use of these nanocarriers increased the stability and reduced the irritating secondary effects commonly observed in vitamin A derivatives. Among the three nanocarriers, liposomes and NEs showed the highest skin permeation. Moreover, the biocompatible properties of liposomes resulted in higher retention in the deeper skin layers, no tissue irritation, and increased skin hydration [20].

Nanotechnology extends even further to environmental applications, particularly in water treatment. The application of nanostructure fibers like polyaniline as a coating for solid phase microextraction (SPME) of organochlorine pesticides in contaminated water samples has shown high extraction efficiency [21]. Another example is the use of $\mathrm{TiO}_{2}$ nanostructures as semiconductor photocatalysts for efficient degradation of organic pollutants (dyes) in contaminated water [22]. The multiple applications of $\mathrm{TiO}_{2}$-based nanotechnology have been extensively reviewed elsewhere [23-25].

\subsection{NANOPARTICLES AND CANCER MEDICINE}

One of the most promising areas of nanotechnology is its application to cancer diagnosis and treatment. Cancer is a group of diseases that figure among the leading causes of death worldwide, with approximately 14 million new cases and 8.2 million deaths per year. Unfortunately, currently available chemotherapeutic agents have the disadvantage of affecting not only tumor cells but also normal cells, and they produce diverse secondary effects such as cardiotoxicity, cytotoxicity, and neurotoxicity [1,26,27]. In the past decade, nanoparticles have been successfully introduced in cancer medicine to overcome the adverse effects associated with the currently used chemotherapeutic agents. The use of nanoparticles as drug carriers has improved treatment efficacy, increased drug stability, and reduced the toxicity 
of classic chemotherapeutic drugs [1,7]. In fact, several commonly used anticancer agents are administered now as nanoliposomal formulations [7-11]. Theoretically, nanoparticles are effective as delivery systems because they can be engineered to be more soluble and have reduced renal clearance and a longer half-life $\left(t_{1 / 2}\right)$ [28]. Furthermore, nanoparticles have shown improved bioavailability due to their enhanced infiltration, greater retention, and high specificity at target disease tissues, which also serves as a protective mechanism for the surrounding healthy tissue [29].

\subsubsection{Common nanoparticles with potential use for cancer treatment and diagnosis}

Nanoparticles as drug and imaging systems provide unique approaches for cancer treatment and diagnosis. Different nanoparticle systems like inorganic and organic nanoparticles have been developed as nanocarriers of molecular cargos such as small molecules, oligonucleotides, peptides, proteins, genes, and imaging agents. Inorganic nanoparticles are primarily metal-based and include semiconductors (quantum dots), silica-based nanoparticles, metallic nanoparticles, magnetic nanoparticles, and fullerene nanoparticles (carbon nanotubes). On the other hand, organic nanoparticles, which range in size from $10 \mathrm{~nm}$ to $1 \mu \mathrm{m}$, mainly include polymer-based nanoparticles (such as polymeric nanoparticles, micelles, and dendrimers) and liposomes.

\subsubsection{Inorganic nanoparticles}

\subsection{Quantum dots}

Quantum dots (2-10 nm) are fluorescent spherical nanoparticles composed of an elemental core encompassed by a metal shell [30]. They are mostly used for biomarker screening and medical imaging. Quantum dots have great advantages over organic dyes and allow tracking activities over a period of time [31]. For example, herceptin-conjugated $\mathrm{CdSe} / \mathrm{ZnS}$ quantum dots (QD-Her) were shown by fluorescence microscopy to have selective internalization into target breast cancer cells through membrane receptors. Following internalization, the release of herceptin to the cytoplasm resulted in the induction of cell death [32]. Unfortunately, quantum dots are synthesized from heavy metals, and therefore their use is limited due to toxicity concerns $[33,34]$.

\subsection{Silica-based nanoparticles}

Silica-based nanoparticles include mesoporous and core shell silica nanoparticles [35]. Mesoporous silica nanoparticles (MSNs) form a complex network of channels through the interior, whereas core shell silica nanoparticles contain surface pores leading to a central cavity. The distribution and size of the pores determine the kinetics of drug release [36,37]. For 
example, Gao et al. used doxorubicin-loaded hollow MSNs with three different pore sizes and found that nanoparticles with a larger pore size exhibited higher cellular uptake as well as faster intracellular drug release [38]. A pore size-dependent anticancer activity against multidrug-resistant (MDR) breast cancer cells was observed [38]. Tanaka et al. showed that incorporation of siRNA against the EphA2 oncoprotein into multistage MSNs with neutral nanoliposomes resulted in sustained gene silencing in mouse models of ovarian cancer [39]. A reduction in tumor burden and decreased angiogenesis and cell proliferation were observed [39]. No observable toxicity associated with the nanoparticles, nanoliposomes, or siRNA was detected [39].

\subsection{Metal-based nanoparticles}

Metal-based nanoparticles, particularly gold, silver, and platinum, have also been investigated as drug delivery systems [40,41]. For example, Qian et al. used gold nanoparticles (AuNPs) conjugated with cetuximab [a monoclonal antibody that targets the epidermal growth factor receptor (EGFR)] and showed that these nanoparticles enhance the cytotoxicity of cetuximab in EGFR-positive non-small-cell lung cancer (NSCLC) both in vitro and in vivo. Cetuximab conjugated with AuNPs significantly suppressed the proliferation and migration, and accelerated apoptosis of high EGFR expressing NSCLC cells compared with cetuximab alone. Moreover, a significant reduction in tumor weight and tumor volume with minimal toxicity was observed in a mouse lung cancer model following treatment with cetuximab-conjugated AuNPs [42].

\subsection{Magnetic nanoparticles}

Magnetic nanoparticles used for drug delivery are made of iron-based crystals, typically magnetite or maghemite. For tissue targeting, a localized magnetic field is generated near the target region using external magnets $[43,44]$. For example, Wen et al. showed that a combination treatment with folic-acid-modified magnetic nanoparticles (FA-MNPs) and a $100 \mathrm{~Hz}$ extremely low-frequency electromagnetic field (generated by using a solenoid coil) decreases proliferation and increases apoptosis of liver cancer cells compared to FA-MNPs alone [45].

\subsection{Carbon-based nanotubes}

Carbon-based nanotubes (1-100 nm) are tubular structures formed by rolling graphene sheets into cylindrical carbon networks [46]. Depending on the number of sheets used, carbon nanotubes can be categorized into single(SWNT) or multi-walled nanotubes (MWNT) [47]. Despite being used in drug delivery, their insolubility and toxicity limit their application $[48,49]$. Nevertheless, these limitations can be overcome by surface modifications that increase biocompatibility [47]. For example, Cao et al. used MWNTs modified with poly(ethyleneimine) (PEI) and conjugated with fluorescein isothiocyanate (FI) and hyualuronic acid (HA) for targeted delivery of doxorubicin to cancer 
cells overexpressing CD44 receptors. Results showed that the carrier material had good biocompatibility at the concentrations tested [50]. In addition, the complexes were able to specifically target cervical carcinoma cells overexpressing CD44, leading to growth inhibition via receptor-mediated binding and intracellular uptake [50].

\subsubsection{Organic nanoparticles}

\subsection{Polymer-based nanoparticles}

Polymer-based nanoparticles include polymeric nanoparticles, micelles, and dendrimers.

\subsection{Polymeric nanoparticles}

Polymeric nanoparticles are colloidal solid particles $(50-300 \mathrm{~nm})$ prepared using synthetic polymers [poly(lactic acid) (PLA), poly(lactic-co-glycolic acid) (PLGA), PEI, and poly(ethylene glycol) (PEG)], synthetic hydrogels [poly(acrylamide)], natural polymers (chitosan), or hydrolytically or enzymatically degradable polymers (collagen) [51]. PLA and PLGA hydrolyze into biologically compatible metabolites, which are eventually eliminated from the body as carbon dioxide and water, and therefore are less cytotoxic [52,53]. The functionality of PLA and PLGA nanoparticles can be improved by surface modifications such as PEGylation, lipid-coating, and cell-targeting ligands [54]. For example, docetaxel-loaded PLA nanoparticles conjugated with a peptide highly specific for binding pulmonary adenocarcinoma tissue resulted in a significant decrease in the metastatic tumor area in the liver of a nude mouse model of lung cancer compared with the absence of the targeting peptide [55].

\subsection{Micelles}

Micelles are amphiphilic shell-core structures able to entrap and carry hydrophobic molecular cargos [56]. Their small size prevents their uptake by the reticulo-endothelial system (RES), increasing circulation time and allowing percutaneous lymphatic transport as well as extravasation from blood vessels to target tumor tissues $[30,57,58]$. Moreover, micelles can be constructed to respond to external stimuli such as changes in temperature, light, and $\mathrm{pH}$ [59]. For example, Sajomsang et al. demonstrated that curcumin encapsulated within $\mathrm{pH}$ responsive polymeric micelles increased the percentage of apoptotic cervical cancer cells compared to free curcumin [60].

\subsection{Dendrimers}

Dendrimers are synthetic, highly branched oligomers that form three-dimensional molecules. They are synthesized in a stepwise manner resulting in an inner core moiety with radially attached branch layers [30,37,61,62]. Drug loading capacity as well as the kinetics of drug release can be altered by changing the number of layers. Further modulation can be achieved by incorporation of degradable linkages between the dendrimer and molecular cargo, such as amide or ester bonds. For example, Khatri et al. used amide- 
-bonded methotrexate conjugated dendrimers and found that the viability of uterine sarcoma cells was decreased in a dose-dependent manner compared to methotrexate alone [63].

\subsection{Liposomes}

Although several nanoparticle systems have been proposed as delivery carriers of therapeutic and diagnostic agents, liposomes - particularly nanoliposomes - are the most frequently used nanoparticles for drug delivery [64]. Liposomes (ranging in size from 30 to $200 \mathrm{~nm}$ ) are vesicular cell membrane-like structures consisting of a phospholipid bilayer that forms by the self-assembly of dissolved lipid molecules [65]. After assembly, liposomes may contain a mixture of small unilamellar vesicles (SUV), large unilamellar vesicles (LUV), multilamellar vesicles (MLV), or multivesicular vesicles (MVV) [66], as shown in Figure 1. Extrusion or sonication methods are normally used to obtain a homogeneous population of SUV [67].

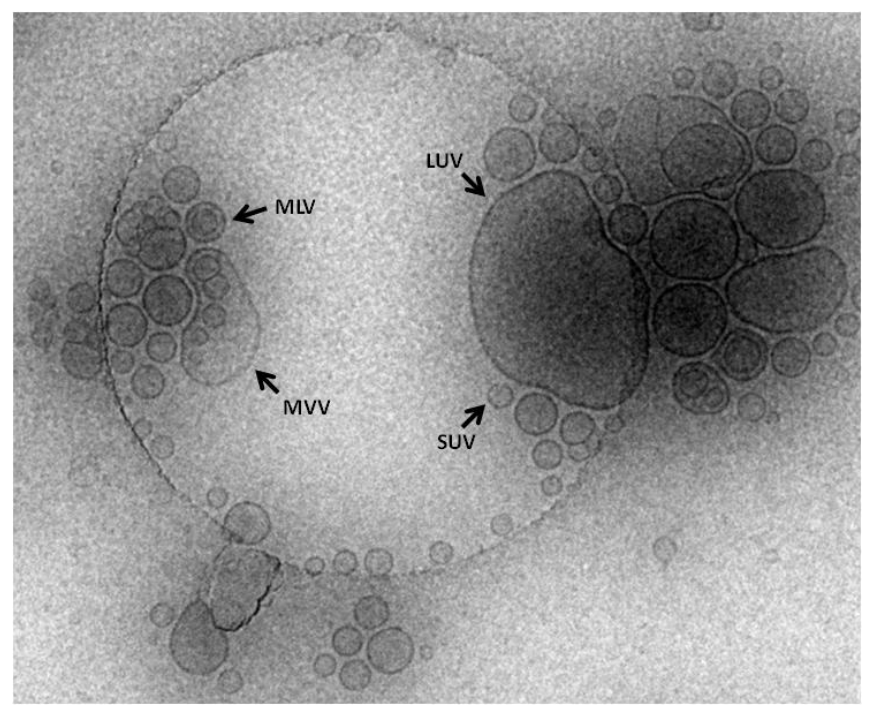

Figure 1. Cryo-electron microscopy profile of DOPC-PEG nanoliposomes

Liposomes may contain natural or synthetic phospholipids [phosphatidylcholine (PC) and phosphatidylethanolamine (PE)] and cholesterol $[7,11,68]$. The phospholipid bilayer is capable of storing both hydrophobic and hydrophilic compounds. Therefore, liposomes are often used as delivery systems for a great variety of molecular cargos. The lipids used can be divided into several categories, including cationic, anionic, neutral, or a mixture of lipids, each one with different charges [66]. The overall charge of the liposomes can be manipulated to enhance interaction with molecules of interest. 


\subsection{Cationic lipids}

Cationic lipids are often formed with a neutral helper lipid such as dioleoylphosphatidylethanolamine (DOPE), which increases stability and enhances cellular uptake [69-71]. The positive charge in cationic lipids [including $N$-[1-(2,3-dioleyloxy)propyl]- $N, N, N$-trimethylammonium chloride (DOTMA) and [1,2-bis(oleoyloxy)-3-(trimethylammonio) propane] (DOTAP)] allows spontaneous interactions with DNA or RNA as well as binding with negatively charged components of the cell membrane, facilitating the entry of molecular cargos into target tissues [66]. However, although cationic lipids interact easily with negatively charged surfaces, toxicity due to activation of pro-apoptotic and pro-inflammatory cascades is a major concern [72].

\subsection{Anionic lipids}

Anionic lipids such as phosphatidic acid (PA), phosphatidylglycerol (PG), and phosphatidylserine (PS) have been investigated as alternatives to cationic lipids. Unfortunately, the negative charge in anionic lipids results in electrostatic repulsions with molecular cargos, limiting their application as nanocarriers [66]. In addition, negatively charged liposomes are taken up faster by macrophages in the blood circulation. Nevertheless, when the target is the phagocyte monocyte system, the use of these liposomes is an advantage [73].

\subsection{Neutral lipids}

Neutral lipids such as dioleoylphosphatidylcholine (DOPC) have been used as a way to circumvent the problems associated with cationic and anionic nanoliposomes. For example, Tekedereli et al. showed that systemic administration of DOPC-nanoliposomes encapsulating Bcl-2-targeted siRNA (NL-Bcl-2-siRNA) leads to anti-tumor activity and growth suppression of estrogen receptor-negative [ER (-)] and ER-positive (+) breast cancer orthotopic xenograft models with no obvious toxicity. Moreover, a combination of NL-Bcl-2-siRNA with doxorubicin increased chemotherapy efficacy in both animal models compared to control groups [74].

\subsubsection{Nanoliposomes as delivery carriers of therapeutic and imaging agents}

As drug carriers, nanoliposomes can be coated with PEG, which prolongs the circulation half-life of the nanocarrier and improves biodistribution. Therefore, PEGylation may be used to reduce uptake within the reticuloendothelial system (RES) and allow higher drug concentrations to be delivered to target tissues before clearance [9]. Also, PEG provides a linker for attachment of targeting moieties such as small-molecule ligands, peptides, and monoclonal antibodies, improving tissue specificity [75]. Nanoliposomes carrying chemotherapeutic drugs such as PEGylated doxorubicin (Doxil and Lipo-Dox), non-PEGylated doxorubicin (Myocet), daunorubicin (DaunoXome), cytarabine (DepoCyt), and vincristine (Marqibo) have been clinically approved for cancer 
therapy [7-11]. Additional liposomal formulations are under clinical investigation [7-11].

Lipid-based nanoparticles have also been combined with imaging agents for medical diagnostics and assessment of treatment efficacy [30,61,76]. Alone, imaging systems such as magnetic resonance imaging (MRI), positron emission tomography (PET), and single photon emission computed tomography (SPECT) suffer from limited resolution or sensitivity [77]. However, nanoliposomes have shown great promise in overcoming these limitations. Imaging agents such as radionuclides can be incorporated into either the bilayer or the interior of the nanoliposome, making it suitable for imaging systems [78-80].

\subsubsection{Nanoliposomes as delivery systems of siRNA and miRNAs for cancer treatment}

Since the discovery of RNA interference (RNAi), small RNA molecules including siRNAs and miRNAs have become a powerful therapeutic modality for targeting genes of interest, including "undruggable targets" [81]. Therefore, among the drugs that are currently under clinical trials, RNAi molecules have emerged as a treatment modality of exceptional promise for cancer and other diseases. SiRNAs are small non-coding RNAs (21-27 nucleotides in length) that bind complementary target messenger RNAs (mRNAs), preventing translation and selectively silencing gene expression [82]. Synthetic siRNAs are the most commonly used structures in RNAi-based therapeutic formulations and are designed to target a single key gene which is generally overexpressed in cancer cells compared with normal cells $[82,83]$. In a more recent modality, miRNAs and anti-miRNA agents are used for RNAi-based therapeutics.

MiRNAs are endogenously expressed small non-coding RNAs (21-25 nucleotides in length) that function as post-transcriptional regulators of gene expression $[84,85]$. Recent evidence indicates that the human genome may encode over 1500 miRNAs, which regulate about $60 \%$ of human genes [7]. Multiple studies involving various types of human cancers have demonstrated that miRNAs have a fundamental role in tumorigenesis, drug resistance, and metastasis [86-94]. While most of these miRNAs are down-regulated and act as tumor suppressor genes, some are up-regulated and may represent novel oncogenes (oncomiRs) [95]. Thus, miRNA-targeted therapies can be used to inhibit the action of oncomiRs by single-stranded RNA molecules complementary to the targeted miRNA (antagomirs) or to activate the function of tumor suppressor genes by partially double-stranded RNA molecules that mimic endogenous precursor miRNAs (miRNA mimics) [7,96].

Despite their potential use in cancer treatment, the major limitations of RNAi-based therapies include off-target effects, poor uptake, degradation, and rapid clearance following administration [6,97-99]. To overcome these barriers, carrier systems such as nanoliposomes have been explored for effective and 
safe delivery of RNAi molecules [100,101]. These nanocarriers protect RNAi molecules from degradation, facilitating uptake by target tissues, which is also affected by the charge of the liposome. In 2005, Landen et al. used neutral DOPC nanoliposomes in ovarian cancer mouse models and found a 10 -fold improvement in the delivery of siRNA compared with cationic DOTAP, and a 30 -fold improvement over naked siRNA. Moreover, incorporation of EphA2-siRNA into DOPC nanoliposomes inhibited tumor growth in ovarian cancer mouse models compared with a negative control siRNA. This effect was further enhanced when using DOPC-encapsulated EphA2-siRNA in combination with paclitaxel [102]. Currently, siRNA-EphA2-DOPC is in a phase I clinical trial (not yet recruiting) for patients with advanced solid tumors [103].

Another example of the use of neutral nanoliposomes in siRNA delivery for cancer treatment includes the encapsulation of survivin splice variant 2B-targeted siRNA (2B-siRNA) into DOPC nanoliposomes. Silencing of survivin $2 \mathrm{~B}$ in taxane-resistant ovarian cancer orthotopic mouse models resulted in a significant reduction in tumor growth, which was further enhanced in combination with docetaxel. Decreased microvessel density (MVD) and cell proliferation was observed, as well as induction of apoptosis in mice treated with 2B-siRNA-DOPC nanoliposomes compared with controls. Silencing of survivin $2 \mathrm{~B}$ resulted in similar growth inhibitory effects as observed when silencing all survivin splice variants simultaneously, proposing 2B-siRNADOPC as a novel and specific anti-survivin therapy in ovarian cancer.

Targeting of the nuclear receptor coregulator PELP1 (proline-, glutamic acid-, leucine-rich protein-1) in ovarian cancer by systemic administration of siRNADOPC nanoliposomes has also been shown to effectively reduce PELP1 expression and significantly reduce tumor growth, metastatic tumor nodules, and ascites volume in xenograft models. Results suggest that PELP1-siRNADOPC nanoliposomes can be used as a potential therapeutic modality for the prevention or treatment of ovarian cancer metastasis [105]. Several other siRNA-liposomal formulations targeting key genes involved in cancer growth, proliferation, drug resistance, and metastasis are under investigation [106110].

In addition to siRNA-EphA2-DOPC, a number of other RNAi lipid nanoparticles have entered clinical trials. For example, Alnylam Pharmaceuticals has completed a phase I dose escalation trial for ALN-VSP02 - a dual-targeted lipid particle siRNA drug formulated against vascular endothelial growth factor (VEGF) and kinesin spindle protein (KSP) - in patients with advanced solid tumors with liver involvement [111]. A phase I dose escalation study sponsored by the National Cancer Institute on hepatic intra-arterial administration of TKM-080301 - a lipid nanoparticle formulation of a siRNA targeting the serine/threonine kinase PLK1 - in patients with primary or secondary liver cancer has also been completed [112]. Further phase I / II dose escalation and phase I dose escalation with phase II expansion cohort studies 
sponsored by Tekmira Pharmaceutical are currently undergoing trial for the determination of safety, tolerability, and pharmacokinetics of TKM-080301 in patients with advanced solid tumors, and preliminary anti-tumor activity of TKM-080301 in patients with advanced hepatocellular carcinoma (HCC), respectively $[113,114]$. Silence Therapeutics has also initiated a phase Ib / IIa study for Atu027 - a siRNA-lipoplex directed against protein kinase N3 (PKN3) - in combination with gemcitabine for treatment of locally advanced or metastatic pancreatic cancer. This study aims to evaluate the potential attenuation of further disease progression by the anti-metastatic effect of Atu027 in combination with the anti-neoplastic activity of gemcitabine [115]. Moreover, phase I and phase Ib/II study trials sponsored by Dicerna Pharmaceuticals are evaluating the safety, tolerance, and maximum dose of Dicer substrate short interfering RNAs (DsiRNAs) in lipid nanoparticles targeting the myelocytomatosis (MYC) oncogene (DCR-MYC) in patients with solid tumors, multiple myeloma, or lymphoma, as well as the recommended phase II dose of DCR-MYC in patients with hepatocellular carcinoma [116,117].

Significant advances have also been made in the development of miRNA-based therapies. For example, systemic administration of an miR-34a mimic complexed with an amphoteric liposomal formulation showed significant growth inhibition in orthotopic models of liver cancer, suggesting its potential use for cancer treatment [118]. In fact, a multicenter phase I trial sponsored by Mirna Therapeutics is currently recruiting participants for safety evaluation of MRX34 - a miR-34 mimic liposomal injection - in patients with primary liver cancer (hepatocellular carcinoma), other solid tumors with liver metastasis, and hematologic malignancies [119].

Innovative gene silencing therapies such as the combination of miRNA and siRNA incorporation into nanoparticles have also been evaluated in ovarian cancer in vitro and in vivo models [120]. In this study, dual inhibition of EphA2 using DOPC-nanoliposomes loaded with miR-520d-3p and EphA2 siRNA resulted in therapeutic synergy and enhanced tumor suppression compared with either therapy alone. Such 'boosting' in anti-tumor effects suggests the feasibility of a new concept of RNAi-based therapy for cancer and other diseases [120].

\subsubsection{Other nanoparticles as delivery systems for therapeutic and imaging agents in cancer}

Another example of a drug delivery system that has shown great promise for tumor targeting and treatment due to its specificity for the affected tissue and low induction of the host's immune system consists of a RNA-based nanoparticle by itself known as packaging RNA (pRNA) [29]. The pRNA (one of the six pRNA subunits of the DNA packaging motor of bacteriophage phi29) is a 117-nucleotide (nt) RNA molecule that can acquire a stable nanoparticle structure of about $11 \mathrm{~nm}$ in size [29]. These RNA-based nanoparticles induce 
low toxicity to healthy organs such as the liver, lungs, and kidneys [121]. Therefore, they are attractive systems for tumor targeting and drug delivery due to their biocompatibility and biodegradability [29].

\subsection{NANOPARTICLE FORMULATIONS FOR THE TREATMENT OF OTHER HUMAN CONDITIONS}

The use of nanoparticles as delivery systems for a variety of drugs and treatments is an emerging area of interest, not only for cancer, but for many other health conditions ranging from bacterial and viral infections to cardiovascular disease. The major concerns of bacterial and fungal infections include the increasing resistance to antibiotics and the systemic side effects associated with the use of antifungal treatments. To overcome these concerns, nanotechnology offers an opportunity to develop drug carrier systems with higher specificity and efficacy. Such nanocarriers offer the possibility of extended release as well as the potential of reducing the efflux of the drug through the transmembrane proteins of the cell wall [122]. An example of a nanocarrier-mediated drug delivery system is the use of liposomal amphotericin B (L-AmB) for the treatment of fungal infections [123].

Liposomal antibiotic formulations are also being developed as aerosols for the treatment of opportunistic lung infections, including infection by Pseudomonas aeruginosa in patients with cystic fibrosis (CF). A phase II study of neutral liposomal amikacin (Arikace) administered once daily by a nebulizer to patients with CF resulted in a decrease of the pseudomonal sputum content and an improvement in the pulmonary function and respiratory symptoms (all of which suggest a longer antimicrobial effect), as well as few or no adverse side effects compared to the placebo [124]. The development of liposomal drug formulations as nebulized treatments for lung infections is promising due to the ability to make neutral liposomes containing dipalmitoylphosphatidylcholine (DPPC) and cholesterol, which are two of the main components of the pulmonary surfactant [125].

Nanostructures are also being used to develop carrier systems for the treatment of viral infections. An example is the development of siRNAs carried in stable nucleic acid-lipid particles (SNALPs) for the treatment of ebola-virus infection [126]. Another example includes a recent study on the development of non-viral vectors for chronic hepatitis $\mathrm{C}$ virus (HCV) treatment, which showed that a preparation of SLNs coated with hyaluronic acid and transporting the plasmid short hairpin RNA 74 (shRNA74) were effective in silencing the internal ribosome entry site of HCV (HCV-IRES), thus inhibiting its viral replication [127]. Another study showed a nanoliposome formulation with short synthetic RNA (sshRNA) SG220 also targeting the HCV-IRES, which yielded increased gene suppression [128]. In addition to nanostructures as vectors for siRNAs, shRNAs, and sshRNAs in HCV treatment, nanostructures 
have been used as delivery systems for antagomirs. For example, anti-miRNA-122 (antagomir-122) complexed with interfering nanoparticles (iNOP-7) resulted in effective delivery into the cytoplasm of hepatocytes and silencing of endogenous miR-122 in HCV-positive mice [129].

Another modality under study includes the use of siRNAs as topical treatments in microbicide formulations to inhibit viral replication of herpes simplex virus type 2 (HSV-2) [130] and human immunodeficiency virus (HIV) type 1 [131] two of the most common causes of sexually transmitted diseases (STDs) worldwide [132]. In fact, the use of nanostructures in topical microbicides is a promising treatment option for STDs. For example, a recent study showed an enhanced anti-viral effect against HIV as a result of the combination of two anionic carbosilane dendrimers (G2-STE16 and G2-S24P). The combination of both dendrimers resulted in an enhanced inability of the HIV viral envelope protein gp120 to bind to the CD4 receptor. These results suggest the use of such dendrimer combinations as topical microbicide formulations to prevent HIV infection through the vaginal mucosa in high-risk populations [133].

The nanotechnological advances in cardiovascular medicine have opened the door for innovative treatments that can reverse heart disease, the leading cause of death in the United States [134]. For instance, a recent study by Serpooshan et al. showed the therapeutic benefit of PEG-based liposome-encapsulated apelin (lipoPEG-PA13) on hypertrophied heart. Apelin - an adipokine that is endogenously found in the human body - is involved in homeostasis regulation of bodily fluids, blood pressure, and heart function. Injection of lipoPEG-PA13 into mice with hypertrophic heart injury led to a reduction in the size of the left ventricle and reduced fibrosis [135]. In addition, lipoPEG-PA13 showed a sustained release in the blood stream of mice compared with mice treated with non-encapsulated apelin [135]. Another therapeutic modality includes the application of siRNAs to target genes involved in lipid metabolism. For example, a phase I study trial of the lipid nanoparticle (LNP)-packaged siRNA drug, ALN-PCS02, proved to be effective in decreasing low-density lipoprotein cholesterol (LDL-C) levels in patients' serum by targeting and inhibiting the enzyme PCSK9 (proprotein convertase subtilisin/kexin type 9). PCSK9 is responsible for the degradation of LDL receptors (LDLR) and subsequent decreased metabolism of LDL-C. Therefore, by inhibiting PCSK9, plasma LDL concentration decreases, thus lowering the risk of cardiovascular disease [136].

\subsection{FUTURE DIRECTIONS AND CHALLENGES}

The use of nanoparticles as carriers for cancer treatment could drastically improve the stability and therapeutic effectiveness of the currently used and newly discovered anticancer agents. Various nanoparticle systems, including silica-based, liposomes, polymeric, and metal nanoparticles, among many 
others, have good biocompatibility, biodegradability, and high encapsulation efficiency. In addition, they are easy to prepare at very low cost, and most of the components in liposomal formulations are already approved by the Food and Drug Administration (FDA). Liposomes have the extra capability to carry both lipophilic and hydrophilic molecules, and they can be functionalized with several ligands, which increase specificity and therapeutic index.

Before proposing nanoparticles as reliable drug delivery systems for cancer treatment and/or imaging, further research should be performed mainly to answer the following important questions:

1) How can the circulation time of the nanoparticle formulations be increased to improve their therapeutic efficacy [137]?

2) Once the nanoparticles come into the blood circulation, how is the nanoparticle surface altered by blood-associated proteins and how can this affect the interaction between the nanoparticles and the target cells $[138,139]$ ?

3) What are the major mechanisms by which nanoparticles interact with the plasma membranes to release their cargo $[140,141]$ ?

4) What amount of nanoparticles and their cargo are truly associated with the tumor tissue relative to other tissues and organs [142]?

5) Once in the tumor tissue, what amount of nanoparticles and/or their content remains in the periphery relative to the amount of cargo effectively released inside the tumor cells [143]?

6) How can $\mathrm{pH}$-sensitive nanoparticles be created to take advantage of $\mathrm{pH}$ differences between normal tissues $(\mathrm{pH}=7.4)$ compared with tumor cells $(\mathrm{pH}=6.0-6.5)[144]$ ?

7) Is the molecular target highly abundant in cancer cells and negligible in normal non-cancerous cells [145-147]?

8) If functionalized nanoparticles (targeted nanoparticles) are being proposed, is the target receptor highly abundant in cancer cells as compared with normal tissue cells [148]?

9) How can nanoparticles with more than one different drug be designed to target multiple intracellular targets in cancer cells [149]?

10) Is the dose/schedule therapy appropriate to avoid the drastic decrease in nanoparticle blood concentration as a result of the accelerated blood clearance phenomena [150]?

The tumor cell heterogeneity is another concern, as the selected molecular target might be expressed only by a small number of cancer cells compared with the enormous genetically different cancer cell populations in the tumor, including cancer stem cell populations [151]. For safety purposes, pharmacokinetics, pharmacodynamics, and tissue distribution studies of the nanoparticle and nanoparticle-cargo formulations are highly desirable [152]. 
Therapies able to cross the blood-brain barrier (BBB) are also needed for brain tumor treatment and/or imaging [153-156].

The use of nanoparticles - particularly nanoliposomes - for the delivery of RNAi-based therapies is a rapidly growing research area. It would appear that this therapeutic modality has immense potential for the treatment of cancer and many other human conditions.

\subsection{CONCLUSIONS}

The use of nanoparticles as drug carriers for therapy and diagnostic agents is slowly moving into the clinic, not only for the treatment of cancer but for many other human conditions as well. However, improved nanoparticles able to deliver drugs to desired sites are still urgently needed. Such systems might significantly increase drug safety and effectiveness. Therefore, it is anticipated that in the near future, more nanoparticle formulations, particularly for the delivery of novel therapies including RNAi-based modalities, will be used clinically.

\section{ACKNOWLEDGEMENTS}

This project was supported partially by institutional seed funds from the University of Puerto Rico Comprehensive Cancer Center (PEVM) and the National Institutes of Health, Minority Biomedical Research Support (MBRS) RISE Grant Number R25GM061838 (JMRG). 


\section{REFERENCES}

1. A.Z. Wang, R. Langer, O.C. Farokhzad. Annu. Rev. Med. 63 (2012) 185-198.

2. N.A. Ochekpe, P.O. Olorunfemi, N.C. Ngwuluka. Trop. J. Pharm. Res. 8(3) (2009) 265-274.

3. M.R. Mozafari, C. Johnson, S. Hatziantoniou, C. Demetzos. J. Liposome Res. 18 (2008) 309-327.

4. N. Moussaoui, M. Cansell, A. Denizot. Int. J. Pharm. 242 (2002) 361-365.

5. I. Gehrke, A. Geiser, A. Somborn-Schulz. Nanotechnol. Sci. Appl. 8 (2015) 1-17.

6. B. Ozpolat, A.K. Sood, G. Lopez-Berestein. Adv. Drug Deliv. Rev. 66 (2014) 110-116.

7. M.R. Díaz, P.E. Vivas-Mejia. Pharmaceuticals (Basel) 6 (2013) 1361-1380.

8. $\quad$ F. Perche, V.P. Torchilin. J. Drug Deliv. (2013) 705265.

9. P.P. Deshpande, S. Biswas, V.P. Torchilin. Nanomedicine (Lond). 8 (2013) 1509-1528.

10. A. Wicki, D. Witzigmann, V. Balasubramanian, J. Huwyler. J. Control. Release 200 (2015) 138-157.

11. Y. Fan, Q. Zhang. Asian J. Pharm. Sci. 8 (2013) 81-87.

12. A.A. Farooqi, Z.U. Rehman, J. Muntane. Onco Targets Ther. 7 (2014) 2035-2042.

13. G. Ozcan, B. Ozpolat, R.L. Coleman, A.K. Sood, G. Lopez-Berestein. Adv. Drug Deliv. Rev. (2015).

14. Y. Zhang, Z. Wang, R.A. Gemeinhart. J. Control. Release 172 (2013) 962-974.

15. T.V. Duncan. J. Colloid Interf. Sci. 363 (2011) 1-24.

16. M.R. De Moura, L.H.C. Mattoso, V. Zucolotto. J. Food Eng. 109 (2012) 520-524.

17. S. Torres-Giner, A. Martinez-Abad, M.J. Ocio, J.M. Lagaron. J. Food Sci. 75 (2010) N69-N79.

18. J. Gomez-Estaca, M.P. Balaguer, R. Gavara, P. Hernandez-Munoz. Food Hydrocoll. 28 (2012) 82-91.

19. J.P. Paiva, B.A.M.C. Santos, D.M. Kibwila, T.C.W. Gonçalves, A.V. Pinto, C.R. Rodrigues, A.C. Leitao, L.M. Cabral, M. De Padula. J. Pharm. Sci. 103 (2014) 2539-2545.

20. B. Clares, A.C. Calpena, A. Parra, G. Abrego, H. Alvarado, J.F. Fangueiro, E.B. Souto. Int. J. Pharm. 473 (2014) 591-598.

21. Z. Gao, W. Li, B. Liu, F. Liang, H. He, S. Yang, C. Sun. J. Chromatogr. A 1218 (2011) 6285-6291.

22. V. Scuderi, G. Impellizzeri, L. Romano, M. Scuderi, G. Nicotra, K. Bergum, A. Irrera, B.G. Svensson, V. Privitera. Nanoscale Res. Lett. 9 (2014) 458.

23. S. Leong, A. Razmjou, K. Wang, K. Hapgood, X. Zhang, H. Wang. J. Memb. Sci. 472 (2014) 167-184.

24. Y. Wang, Y. He, Q. Lai, M. Fan. J. Environ. Sci. 26 (2014) 2139-2177.

25. Q. Zhou, Z. Fang, J. Li, M. Wang. Microporous Mesoporous Mater. 202 (2015) 22-35.

26. U.B. Chaudhary, J.R. Haldas. Drugs 63 (2003) 1565-1577.

27. S.E. Lipshultz, S.R. Lipsitz, S.M. Mone, A.M Goorin, S.E. Sallan, S.P. Sanders, E.J. Orav, R.D. Gelber, S.D. Colan. N. Engl. J. Med. 332 (1995) 1738-1743.

28. R.A. Petros, J.M. DeSimone. Nat. Rev. Drug Discov. 9 (2010) 615-627.

29. S. Abdelmawla, S. Guo, L. Zhang, S.M. Pulukuri, P. Patankar, P. Conley, J. Trebley, P. Guo, Q.X. Li. Mol. Ther. 19 (2011) 1312-1322. 
30. N. Bhandare, A. Narayana. J. Nucl. Med. Radiat. Ther. 5(4) (2014) 195.

31. A.M. Smith, S. Dave, S. Nie, L. True, X. Gao. Expert Rev. Mol. Diagn. 6 (2006) 231-244.

32. S.-J. Han, P. Rathinaraj, S.-Y. Park, Y.K. Kim, J.H. Lee, I.-K. Kang, J.-S. Moon, J.G. Winiarz. Biomed. Res. Int. (2014) 954307.

33. M. Fang, C.-W. Peng, D.-W. Pang, Y. Li. Cancer Biol. Med. 9 (2012) 151-163.

34. R. Hardman. Environ. Health Perspect. 114 (2006) 165-172.

35. X. Wu, M. Wu, J.X. Zhao. Nanomedicine 10 (2014) 297-312.

36. M.A. Hayat, Tumors of the Central Nervous System, Volume 5: Astrocytomas, Hemangioblastomas, and Gangliogliomas. Springer Science \& Business Media, Dordrecht, Netherlands, 2012, pp. 1-290.

37. A.H. Faraji, P. Wipf. Bioorg. Med. Chem. 17 (2009) 2950-2962.

38. Y. Gao, Y. Chen, X. Ji, X. He, Q. Yin, Z. Zhang, J. Shi, Y. Li. ACS Nano. 5 (2011) 9788-9798.

39. T. Tanaka, L.S. Mangala, P.E. Vivas-Mejia, R. Nieves-Alicea, A.P. Mann, E. Mora, H.D. Han, M.M. Shahzad, X. Liu, R. Bhavane, J. Gu, J.R. Fakhoury, C. Chiappini, C. Lu, K. Matsuo, B. Godin, R.L. Stone, A.M. Nick, G. Lopez-Berestein, A.K. Sood, M. Ferrari. Cancer Res. 70 (2010) 3687-3696.

40. G. Doria, J. Conde, B. Veigas, L. Giestas, C. Almeida, M. Assunção, J. Rosa, B.V. Baptista. Sensors 12 (2012) 1657-1687.

41. M. Yamada, M. Foote, T.W. Prow. Wiley Interdiscip. Rev. Nanomed. Nanobiotechnol. 7(3) (2014) 428-445.

42. Y. Qian, M. Qiu, Q. Wu, Y. Tian, Y. Zhang, N. Gu, S. Li, L. Xu, R. Yin. Sci. Rep. 4(7490) (2014) 1-8.

43. C. Li, L. Li, A.C. Keates. Oncotarget. 3 (2012) 365-370.

44. M.V. Yigit, A. Moore, Z. Medarova. Pharm. Res. 29 (2012) 1180-1188.

45. J. Wen, S. Jiang, Z. Chen, W. Zhao, Y. Yi, R. Yang, B. Chen. Int. J. Nanomedicine 9 (2014) 2043-2050.

46. M. Zhang, J. Li. Mater. Today 12 (2009) 12-18.

47. V. Rastogi, P. Yadav, S.S. Bhattacharya, A.K. Mishra, N. Verma, A. Verma, J.K. Pandit. J. Drug Deliv. (2014) 670815.

48. S. Fiorito, A. Serafino, F. Andreola, A. Togna, G. Togna. J. Nanosci. Nanotechnol. 6 (2006) 591-599.

49. X. Li, L. Wang, Y. Fan, Q. Feng, F. Cui. J. Nanomater. (2012) 1-19.

50. X. Cao, L. Tao, S. Wen, W. Hou, X. Shi. Carbohydr. Res. 405 (2015) 70-77.

51. C.M. Dawidczyk, L.M. Russell, P.C. Searson. Front Chem. 2 (2014) 69.

52. H.K. Makadia, S.J. Siegel. Polymers (Basel) 3 (2011) 1377-1397.

53. I. Armentano, M. Dottori, E. Fortunati, S. Mattioli, J.M. Kenny. Polym. Degrad. Stab. 95 (2010) 2126-2146.

54. H. Sah, L.A. Thoma, H.R. Desu, E. Sah, G.C. Wood. Int. J. Nanomedicine 8 (2013) 747-765.

55. N. Yang, Y. Jiang, H. Zhang, B. Sun, C. Hou, J. Zheng, Y. Liu, P. Zuo. Mol. Pharm. 12 (2015) 232-239.

56. A.M. Jhaveri, V.P. Torchilin. Front Pharmacol. 5(77) (2014) 1-26.

57. C. Oerlemans, W. Bult, M. Bos, G. Storm, J.F.W. Nijsen, W.E. Hennink. Pharm. Res. 27 (2010) 2569-2589.

58. V. Trubetskoy. Adv. Drug Deliv. Rev. 37 (1999) 81-88.

59. N. Rapoport. Prog. Polym. Sci. 32 (2007) 962-990. 
60. W. Sajomsang, P. Gonil, S. Saesoo, U.R. Ruktanonchai, W. Srinuanchai, S. Puttipipatkhachorn. Int. J. Pharm. 477 (2014) 261-272.

61. A. Sharma, N. Jain, R. Sareen. Biomed. Res. Int. (2013) 960821.

62. V. López-Dávila, A.M. Seifalian, M. Loizidou. Curr. Opin. Pharmacol. 12 (2012) 414-419.

63. S. Khatri, N.G. Das, S.K. Das. J. Pharm. Bioallied Sci. 6 (2014) 297-302.

64. A.S. Abreu, E.M. Castanheira, M.-J.R. Queiroz, P.M. Ferreira, L.A. Vale-Silva, E. Pinto. Nanoscale Res. Lett. 6 (2011) 482.

65. B. Yu, R.J. Lee, L.J. Lee. Methods Enzymol. 465 (2009) 129-141.

66. D.A. Balazs, W. Godbey. J. Drug Deliv. (2011) 326497.

67. A. Puri, K. Loomis, B. Smith, J.-H. Lee, A. Yavlovich, E. Heldman, R. Blumenthal. Crit. Rev. Ther. Drug Carrier Syst. 26 (2009) 523-580.

68. Y. Malam, M. Loizidou, A.M. Seifalian. Trends Pharmacol. Sci. 30 (2009) 592-599.

69. L. Wasungu, D. Hoekstra. J. Control. Release 116 (2006) 255-264.

70. H. Farhood, N. Serbina, L. Huang. Biochim. Biophys. Acta 1235 (1995) 289-295.

71. G. Shim, M.-G. Kim, J.Y. Park, Y.-K. Oh. Asian J. Pharm. Sci. 8 (2013) 72-80.

72. C. Lonez, M. Vandenbranden, J.-M. Ruysschaert. Adv. Drug Deliv. Rev. 64 (2012) 1749-1758.

73. C. Kelly, C. Jefferies, S.-A. Cryan. J. Drug Deliv. (2011) 727241.

74. I. Tekedereli, S.N. Alpay, U. Akar, E. Yuca, C. Ayugo-Rodriguez, H.-D. Han, A.K. Sood, G. Lopez-Berenstein, B. Ozpolat. Mol. Ther. Nucleic Acids 2 (2013) e121.

75. R.R. Sawant, V.P. Torchilin. AAPS J. 14 (2012) 303-315.

76. A.D. Miller. J. Drug Deliv. (2013) 165981.

77. Y. Xing, J. Zhao, P.S. Conti, K. Chen. Theranostics 4 (2014) 290-306.

78. B. Goins, A. Bao, W.T. Phillips. Methods Mol. Biol. 606 (2010) 469-491.

79. A. Srivatsan, X. Chen. Adv. Cancer Res. 124 (2014) 83-129.

80. G. Ting, C.-H. Chang, H.-E. Wang, T.-W. Lee. J. Biomed. Biotechnol. (2010).

81. S.Y. Wu, G. Lopez-Berestein, G.A. Calin, A.K. Sood. Sci. Transl. Med. 6(240) (2014) 240ps7.

82. R. Kanasty, J.R. Dorkin, A. Vegas, D. Anderson. Nat. Mater. 12 (2013) 967-977.

83. S.M. Elbashir. Genes Dev. 15 (2001) 188-200.

84. R.C. Lee, R.L. Feinbaum, V. Ambros. Cell 75 (1993) 843-854.

85. B. Bartel. Nat. Struct. Mol. Biol. 12 (2005) 569-571.

86. G. Di Leva, C.M. Croce. Front Oncol. 3 (2013) 153.

87. S. Saini, S. Majid, S. Yamamura, L. Tabatabai, S.O. Suh, V. Shahryari, Y. Chen, G. Deng, Y. Tanaka, R. Dahiya. Clin. Cancer Res. 17 (2011) 5287-5298.

88. Y. Zhang, A. Dutta, R. Abounader. Front Biosci. 17 (2012) 700-712.

89. P. Olson, J. Lu, H. Zhang, A. Shai, M.G. Chun, Y. Wang, S.K. Libutti, E.K. Nakakura, T.R. Golub, D. Hanahan. Genes Dev. 23 (2009) 2152-2165.

90. M. Rivera-Díaz, M.A. Miranda-Román, D. Soto, M. Quintero-Aguilo, H. Ortiz-Zuazaga, M.J. Marcos-Martinez, P.E. Vivas-Meíja. Am. J. Cancer Res. 5 (2015) 201-218.

91. I.M. Echevarría-Vargas, F. Valiyeva, P.E. Vivas-Mejía. PLoS One 9 (2014) e97094.

92. Y. Wang, S. Kim, I.-M. Kim. Front Oncol. 4 (2014) 143.

93. M.T.M. Van Jaarsveld, J. Helleman, E.M.J.J. Berns, E.A.C. Wiemer. Int. J. Biochem. Cell Biol. 42 (2010) 1282-1290. 
94. G. Misso, M.T. Di Martino, G. De Rosa, A.A. Farooqi, A. Lombardi, V. Campani, M.R. Zarone, A. Gulla, P. Tagliaferri, P. Tassone, M. Caraglia. Mol. Ther. Nucleic Acids 3 (2014) e194.

95. B. Zhang, X. Pan, G.P. Cobb, T.A. Anderson. Dev. Biol. 302 (2007) 1-12.

96. M.S. Nicoloso, R. Spizzo, M. Shimizu, S. Rossi, G.A. Calin. Nat. Rev. Cancer 9 (2009) 293-302.

97. D. Bumcrot, M. Manoharan, V. Koteliansky, D.W.Y. Sah. Nat. Chem. Biol. 2 (2006) 711-719.

98. L. Aagaard, J.J. Rossi. Adv. Drug Deliv. Rev. 59 (2007) 75-86.

99. C.V. Pecot, G.A. Calin, R.L. Coleman, G. Lopez-Berestein, A.K. Sood. Nat. Rev. Cancer 11 (2011) 59-67.

100. C. Xu, J. Wang. Asian J. Pharm Sci. 10(1) (2014) 1-12.

101. P. Kesharwani, V. Gajbhiye, N.K. Jain. Biomaterials 33 (2012) 7138-7150.

102. C.N. Landen, A. Chavez-Reyes, C. Bucana, R. Schmandt, M.T. Deavers, G. Lopez-Berestein, A.K. Sood. Cancer Res. 65 (2005) 6910-6918.

103. EphA2 Gene Targeting Using Neutral Liposomal Small Interfering RNA Delivery, https://clinicaltrials.gov/ct2/show/NCT01591356?term=siRNAEphA2-DOPC\&rank=1 (Feb 16, 2015).

104. P.E. Vivas-Mejia, C. Rodriguez-Aguayo, H.-D. Han, M.M.K. Shahzad, F. Valiyeva, M. Shibayama, A. Chavez-Reyes, A.K. Sood, G. Lopez-Berestein. Clin. Cancer Res. 17 (2011) 3716-3726.

105. D. Chakravarty, S.S. Roy, C.R. Babu, R. Dandamudi, T.J. Curiel, P.E. Vivas-Mejia, G. Lopez-Berestein, A.K. Sood, R.K. Vadlamudi. Clin. Cancer Res. 17 (2011) 2250-2259.

106. Q. Zhu, C. Feng, W. Liao, Y. Zhang, S. Tang. Cancer Cell Int. 13 (2013) 65.

107. L.S. Mangala, V. Zuzel, R. Schmandt, E.S. Leshane, J.B. Halder, G.N. Armaiz-Pena, A. W.A. Spannuth, T. Tanaka, M.M.K. Shahzad, Y.G. Lin, A.M. Nick, C.G. Danes, J.-W. Lee, N.B. Jennings, P.E. Vivas-Mejia, J.K. Wolf, R.L. Coleman, Z.H. Siddik, G. Lopez-Berestein, S. Lutsenko, A.K. Sood. Clin. Cancer Res. 15 (2009) 3770-3780.

108. M.M.K. Shahzad, C. Lu, J.-W. Lee, R.L. Stone, R. Mitra, L.S. Mangala, Y. Lu, K.A. Baggerly, C.G. Danes, A.M. Nick, J. Halder, H.-S. Kim, P. Vivas-Mejia, C.N. Landen, G. Lopez-Berestein, R.L. Coleman, A.K. Sood. Cancer Biol. Ther. 8 (2009) 1027-1034.

109. G. Shim, H.-W. Choi, S. Lee, J. Choi, Y.H. Yu, D.-E. Park, Y. Choi, C.-W. Kim, Y.-K. Oh. Mol. Ther. 21 (2013) 816-824.

110. A.M. Nick, R.L. Stone, G. Armaiz-Pena, B. Ozpolat, I. Tekedereli, W.S. Graybill, C.N. Landen, G. Villares, P. Vivas-Mejia, J. Bottsford-Miller, H.S. Kim, J.-S. Lee, S.M. Kim, K.A. Baggerly, P.T. Ram, M.T. Deavers, R.L. Coleman, G. Lopez-Berestein, A.K. Sood. J. Natl. Cancer Inst. 103 (2011) 1596-1612.

111. Dose Escalation Trial to Evaluate the Safety, Tolerability, Pharmacokinetics and Pharmacodynamics of Intravenous ALN-VSP02 In Patients with Advanced Solid Tumors with Liver Involvement, https://clinicaltrials.gov/ct2/show/NCT00882180?term=ALNVSP02\&rank=1 (Feb 16, 2015).

112. TKM 080301 for Primary or Secondary Liver Cancer, https://clinicaltrials.gov/ct2/show/NCT01437007?term=TKM080301\&rank=2 (Feb 16, 2015). 
113. Safety, Pharmacokinetics and Preliminary Anti-Tumor Activity of Intravenous TKM-080301 in Subjects With Advanced Hepatocellular, https://clinicaltrials.gov/ct2/show/NCT02191878?term=TKM080301\&rank=3 (Feb 16, 2015).

114. A Study to Determine Safety, Pharmacokinetics and Pharmacodynamics of Intravenous TKM 080301 in Neuroendocrine Tumors (NET) and Adrenocortical Carcinoma (ACC), https://clinicaltrials.gov/ct2/show/NCT01262235?term=TKM080301\&rank=1 (Feb 16, 2015).

115. Atu027 Plus Gemcitabine in Advanced or Metastatic Pancreatic Cancer (Atu027-I-02), https://clinicaltrials.gov/ct2/show/NCT01808638?term=Atu027\&rank=1 (Feb 16, 2015).

116. Phase I, Multicenter, Dose Escalation Study of DCR-MYC in Patients with Solid Tumors, Multiple Myeloma, or Lymphoma, https://clinicaltrials.gov/ct2/show/NCT02110563?term=dcr-myc\&rank=1 (Feb 16, 2015).

117. Phase Ib/2, Multicenter, Dose Escalation Study of DCR-MYC in Patients with Hepatocellular Carcinoma, https://clinicaltrials.gov/ct2/show/NCT02314052?term=dcr-myc\&rank=2 (Feb 16, 2015).

118. C.L. Daige, J.F. Wiggins, L. Priddy, T. Nelligan-Davis, J. Zhao, D. Brown. Mol. Cancer Ther. 13 (2014) 2352-2360.

119. A Multicenter Phase I Study of MRX34, MicroRNA miR-RX34 Liposomal Injection, https://clinicaltrials.gov/ct2/show/NCT01829971 (Feb 16, 2015).

120. M. Nishimura, E.-J. Jung, M.Y. Shah, C. Lu, R. Spizzo, M. Shimizu, H.D. Han, C. Ivan, S. Rossi, X. Zhang, M.S. Nicoloso, S.Y. Wu, M.I. Almeida, J. BottsfordMiller, C.V. Pecot, B. Zand, K. Matsuo, M.M. Shahzad, N.B. Jennings, C. Rodriguez-Aguayo, G. Lopez-Berestein, A.K. Sood, G.A. Calin. Cancer Discov. 3 (2013) 1302-1315.

121. Y. Shu, F. Haque, D. Shu, W. Li, Z. Zhu, M. Kotb, Y. Lyubchenko, P. Guo. RNA 19 (2013) 767-777.

122. X. Zhu, A.F. Radovic-Moreno, J. Wu, R. Langer, J. Shi. Nano Today 9 (2014) 478-498.

123. J.A. Maertens, L. Madero, A.F. Reilly, T. Lehrnbecher, A.H. Groll, H.S. Jafri, M. Green, J.J. Nania, M.R. Bourque, B.A. Wise, K.M. Strohmaier, A.F. Taylor, N.A. Kartsonis, J.W. Chow, C.A. Arndt, B.E. DePauw, T.J. Walsh. Pediatr. Infect. Dis. J. 29 (2010) 415-420.

124. J.P. Clancy, L. Dupont, M.W. Konstan, J. Billings, S. Fustik, C.H. Goss, J. Lymp, P. Minic, A.L. Quittner, R.C. Rubenstein, K.R. Young, L. Saiman, J.L. Burns, J.R.W. Govan, B. Ramsey, R. Gupta. Thorax 68 (2013) 818-825.

125. E.J. Veldhuizen, H.P. Haagsman. Biochim. Biophys. Acta 1467 (2000) 255-270.

126. T.W. Geisbert, A.C.H. Lee, M. Robbins, J.B. Geisbert, A.N. Honko, V. Sood, J.C. Johnson, S. de Jong, I. Tavakoli, A. Judge, L.E. Hensley, I. MacLachlan. Lancet 375 (2010) 1896-1905.

127. J. Torrecilla, A. Del Pozo-Rodríguez, P.S. Apaolaza, M.Á. Solinís, A. RodríguezGascón. Int. J. Pharm. 479 (2014) 181-188. 
128. A. Dallas, H. Ilves, J. Shorenstein, A. Judge, R. Spitler, C. Contag, S.P. Wong, R.P. Harbottle, I. Maclachlan, B.H. Johnston. Mol. Ther. Nucleic Acids 2 (2013) e123.

129. J. Su, H. Baigude, J. McCarroll, T.M. Rana. Nucleic Acids Res. 39 (2011) e38.

130. D. Palliser, D. Chowdhury, Q.-Y. Wang, S.J. Lee, R.T. Bronson, D.M. Knipe, J. Lieberman. Nature 439 (2006) 89-94.

131. L.A. Wheeler, V. Vrbanac, R. Trifonova, M.A. Brehm, A. Gilboa-Geffen, S. Tanno, L.D. Greiner, A.D Luster, A.M. Tager, J. Lieberman. Mol. Ther. 21 (2013) 1378-1389.

132. L.A. Wheeler. Infect. Dis. Obstet. Gynecol. (2014) 125087.

133. D. Sepúlveda-Crespo, R. Lorente, M. Leal, R. Gómez, F.J. De la Mata, J.L. Jiménez, M.A. Muñoz-Fernandez. Nanomedicine 10 (2014) 609-618.

134. D.L. Hoyert, J. Xu. Natl. Vital Stat. Rep. 61 (2012) 1-51.

135. V. Serpooshan, S. Sivanesan, X. Huang, M. Mahmoudi, A.V. Malkovskiy, M. Zhao, M. Inayathullah, X.J. Zhang, S. Metzler, D. Bernstein, J.C. Wu, P. Ruiz-Lozano, J. Rajadas. Biomaterials 37 (2015) 289-298.

136. K. Fitzgerald, M. Frank-Kamenetsky, S. Shulga-Morskaya, A. Liebow, B.R. Bettencourt, J.E. Sutherland. Lancet 383 (2014) 60-68.

137. J.-W. Yoo, E. Chambers, S. Mitragotri. Curr. Pharm. Des. 16 (2010) 2298-2307.

138. J. Lazarovits, Y.Y. Chen, E.A. Sykes, W.C.W. Chan. Chem. Commun. (Camb). 51 (2014) 2756-2767.

139. R.M. Pearson, V.V. Juettner, S. Hong. Front Chem. 2 (2014) 108.

140. A. Kumari, S.K. Yadav. Expert Opin. Drug Deliv. 8 (2011) 141-151.

141. C.M. Beddoes, C.P. Case, W.H. Briscoe. Adv. Colloid Interface Sci. 218C (2015) 48-68.

142. X.-J. Liang, C. Chen, Y. Zhao, P.C. Wang. Methods Mol. Biol. 596 (2010) 467-488.

143. C.L. Waite, C.M. Roth. Crit. Rev. Biomed. Eng. 40 (2012) 21-41.

144. K.B. Sutradhar, M.L. Amin. ISRN Nanotechnol. 2014 (2014) 1-12.

145. S. Basu, P. Chaudhuri, S. Sengupta. Cell Cycle 8 (2009) 3480-3487.

146. C. Huang, M. Li, C. Chen, Q. Yao. Expert Opin. Ther. Targets. 12 (2008) 637-645.

147. V. Wang, W. Wu. BioDrugs 23 (2009) 15-23.

148. S. Xu, B.Z. Olenyuk, C.T. Okamoto, S.F. Hamm-Alvarez. Adv. Drug Deliv. Rev. 65 (2013) 121-138.

149. C.-M.J. Hu, L. Zhang. Biochem. Pharmacol. 83 (2012) 1104-1111.

150. A.S. Abu Lila, H. Kiwada, T. Ishida. J. Control. Release 172 (2013) 38-47.

151. Y.H. Bae, K. Park. J. Control. Release 153 (2011) 198-205.

152. N. Durán, S.S. Guterres, O.L. Alves, V. Zucolotto, Nanotoxicology: Materials, Methodologies, and Assessments. Springer Science \& Business Media; New York, USA, 2014, pp. 1-411.

153. S.R. Hwang, K. Kim. Arch. Pharm. Res. 37 (2014) 24-30.

154. M. Masserini. ISRN Biochem. 2013 (2013) 1-8.

155. A. Domínguez, B. Suárez-Merino, F. Goñi-de-Cerio. J. Nanosci. Nanotechnol. 14 (2014) 766-779.

156. M. Tajes, E. Ramos-Fernández, X. Weng-Jiang, M. Bosch-Morató, B. Guivernau, A. Eraso-Pichot, B. Salvador, X. Fernàndez-Busquets, J. Roquer, F.J. Muñoz. Mol. Membr. Biol. 31 (2014) 152-167. 